\section{Perfil lipídico e estado nutricional de adolescentes}

\author{
Blood lipid levels and nutritional \\ status of adolescents
}

Danielle Franklin de Carvalho'

Adriana de Azevedo Paiva'

Adriana Suely de Oliveira Melo'

Alessandra Teixeira Ramos ${ }^{2}$

Josimar dos Santos Medeiros ${ }^{2}$

Carla Campos Muniz de Medeiros'

Maria Aparecida Alves Cardoso

${ }^{1}$ Núcleo de Estudos e Pesquisas Epidemiológicas (NEPE), Universidade Estadual da Paraíba.

${ }^{2}$ Departamento de Farmácia, Universidade Estadual da Paraíba.

Correspondência: Danielle Franklin de Carvalho. Rua XV de Novembro, 189 - Palmeira - Campina Grande/PB CEP: 58102-300. E-mail: daniellefranklin6@gmail.com

\section{Resumo}

Objetivos: Avaliar a associação entre obesidade e dislipidemias em adolescentes do ensino público e privado de Campina Grande-PB, Brasil. Métodos: Estudo transversal com 180 adolescentes de 14 a 17 anos matriculados no ensino público e privado de Campina Grande-PB. O estado nutricional foi classificado segundo os percentis do Índice de Massa Corporal para sexo e idade. Foi realizada coleta sangüínea para avaliação do perfil lipídico (colesterol total, frações HDL-colesterol, LDL-colesterol e triglicerídeos). Considerou-se dislipidemia a existência de alteração em pelo menos um dos exames. As análises estatísticas foram realizadas a partir do Epi Info 3.3 e Stata 7.0. Resultados: A prevalência de sobrepeso foi de $14,4 \%$ enquanto que $83,9 \%$ dos estudantes eram eutróficos e 1,7\% apresentavam baixo peso. Não foram encontradas diferenças estatisticamente significantes para o estado nutricional quando estratificado por sexo e tipo de escola. Todas as taxas bioquímicas investigadas mostraram algum nível de alteração. Chamou à atenção a prevalência de dislipidemia, observada em $66,7 \%$ dos estudantes, e a alteração do HDL-colesterol, verificada em $56,7 \%$ destes. Registrou-se associação estatisticamente significante $(\mathrm{p}<0,05)$ do Índice de Massa Corporal, categorizado em tercis, com o colesterol total e sua fração LDL, inclusive quando estratificados por sexo e tipo de escola. Conclusões: Considerando-se a faixa etária estudada, foram elevados os achados de sobrepeso e dislipidemia. Recomenda-se a adição de medidas preventivas, a fim de evitar que cada vez mais crianças e adolescentes venham a se tornar adultos portadores de obesidades e outras doenças crônicas.

Palavras-chave: Adolescente. Estado nutricional. Sobrepeso. Dislipidemia. Hiperlipidemia. 
Abstract

Objective: To evaluate the association between obesity and dyslipidemia in adolescents from public and private schools in Campina Grande, PB, Brazil. Methods: Cross-sectional study of 180 adolescents, aged between 14 and 17 years, enrolled in the public and private school system of Campina Grande-PB. The nutritional status was classified according to the percentiles of Body Mass Index for gender and age. A blood sample was taken to evaluate the lipid profile (total cholesterol, HDLcholesterol, LDL-cholesterol, and triglyceride levels). Dyslipidemia was considered when at least one of the blood parameters exceeded reference limits. Statistical analysis was performed with Epi Info 3.3 and Stata 7.0. Results: The prevalence of overweight was $14.4 \%$, while $83.9 \%$ of students were normal and $1.7 \%$ presented low weight. The nutritional status, when stratified by gender and type of school, did not show statistically significant differences. Dyslipidemia was present in $66.7 \%$ of students and inadequate levels of HDL-cholesterol in $56.7 \%$ of them. The tertiles of Body Mass Index were associated with total cholesterol and the LDL-cholesterol fraction, even when stratified by gender and type of school. Conclusions: Considering the age range of the sample, the results for overweight and dyslipidemias are of concern. There is a need to implement more effective policies oriented towards these adolescents to prevent children and adolescents becoming obese adults with other chronic diseases.

Keywords: Adolescent. Nutritional status. Overweight. Dyslipidemia. Hyperlipidemia.

\section{Introdução}

O excesso de peso e as dislipidemias já estão estabelecidos na literatura científica como fatores determinantes para o desenvolvimento de doenças cardiovasculares ${ }^{1-3}$. No Brasil, estas doenças constituem a principal causa de morbimortalidade ${ }^{4}$.

Estudos mostram a associação entre doença arterial coronariana (DAC) com concentrações séricas elevadas de CT (colesterol total) e LDL-colesterol (low density lipoprotein, cholesterol), e também tem sido demonstrada a relação entre DAC e concentrações séricas reduzidas de HDL-colesterol (high density lipoprotein, cholesteroI $)^{2,5-7}$. A participação dos triglicerídeos na aterogênese ainda permanece em discussão, porém se justifica a dosagem sérica de rotina desse lípide devido à sua freqüente vinculação à doença cardiovascular aterosclerótica e a outros distúrbios metabólicos ${ }^{8}$.

A aterogênese é um processo que tem início na infância, com a formação de estrias gordurosas precursoras das placas ateroscleróticas. Estas começam a surgir na aorta a partir dos 03 anos de idade e, na adolescência, passam a atingir as coronárias, progredindo subseqüentemente nas outras fases do ciclo vital ${ }^{9,10}$. Este processo tende a ser potencializado no decorrer da vida pela obesidade e outros fatores, como história familiar, inatividade física, tipo de dieta e hipertensão $\operatorname{arterial}^{11}$.

Quando tem início nas primeiras fases da vida, a obesidade tende a permanecer ou se agravar com o avançar da idade ${ }^{12}$. Estudos epidemiológicos do perfil lipídico de crianças e adolescentes mostram que o nível de colesterol na infância é um fator preditivo do nível de colesterol na idade adulta $^{13,14}$. A associação entre os dois distúrbios justifica o início da prevenção desde a infância e a adolescência. No entanto, a maioria dos estudos enfoca a problemática apenas na infância ou na idade adulta. A revisão da literatura através do Scielo, utilizando os descritores "dislipidemias" e "hiperlipidemias", mostrou a 
existência de vários estudos descritivos sobre obesidade e perfil lipídico no país, porém são raros aqueles que tratam da associação entre obesidade e dislipidemias na faixa etária aqui abordada, o que indica que a população adolescente ainda não foi devidamente investigada. Na região Nordeste do Brasil, em particular, não foi encontrado qualquer estudo que apresentasse estas características.

Apesar de os estudos a respeito da influência do nível socioeconômico no perfil lipídico não serem consensuais, algumas pesquisas sugerem variações nas alterações lipídicas entre populações de diferentes faixas de renda ${ }^{4,15}$. Além disso, apesar de não representar uma causalidade direta, admite-se que o nível socioeconômico pode interferir no estilo de vida do indivíduo e, portanto, ser um importante fator na determinação da obesidade. Esta também é uma questão que merece maiores estudos, considerando que a obesidade é hoje uma epidemia progressiva nas diferentes faixas etárias e estratos socioeconômicos ${ }^{16}$.

Desta forma, este estudo teve como objetivo avaliar a associação entre obesidade e dislipidemias em adolescentes do ensino público e privado de Campina Grande-PB, Brasil.

\section{Métodos}

Estudo transversal desenvolvido entre fevereiro e maio de 2004. Foram selecionados adolescentes, de ambos os sexos, com faixa etária de 14 a 17 anos, matriculados em uma escola pública e outra privada do município de Campina GrandePB. A escolha da faixa etária baseou-se no pressuposto de que o estirão puberal do crescimento tem início por volta dos $11 \mathrm{e}$ 13 anos, respectivamente, para meninas e meninos ${ }^{17}$. Para assegurar que todos os alunos estudados tivessem ultrapassado o início da puberdade, foi respeitado o intervalo de pelo menos um ano.

Foram selecionados estudantes do ensino público e privado, com o objetivo de avaliar possíveis diferenças nas prevalências de dislipidemia e de sobrepeso/obesidade atribuíveis a condições socioeconômicas. No município existem três escolas privadas de classe media alta. Destas, apenas uma permitiu a realização da pesquisa, particularmente devido à necessidade de coleta de sangue. Seguindo-se os critérios de semelhança com a escola privada, em termos de número de alunos na faixa etária estudada, turnos de aulas e viabilidade de acesso para a coleta de dados, foi selecionada uma escola pública da área urbana do município.

Uma vez definidas as escolas, o estudo foi realizado de acordo com as diretrizes éticas da pesquisa com seres humanos, recomendadas pelo Conselho Nacional de Pesquisa (CONEP) ${ }^{18}$, cujo projeto foi aprovado pelo Comitê de Ética em Pesquisa da Universidade Estadual da Paraíba. Assim, a amostra foi constituída por 85 alunos da escola privada e 95 da pública, correspondendo a todos os adolescentes da faixa etária pré-determinada que apresentaram a autorização dos pais por meio da assinatura de Termo de Consentimento Livre e Esclarecido, que detalhava os objetivos, procedimentos e as etapas do estudo. Foi registrada uma taxa de recusa de $41,0 \%(n=125)$, corroborando as previsões iniciais da pesquisa.

Como instrumentos de coleta de dados foram utilizados um formulário para registro dos dados socioeconômicos, demográficos e das medidas antropométricas dos estudantes; uma balança mecânica tipo plataforma, com capacidade para até 160 Kg e sensibilidade de 100 g (marca Filizola); um antropômetro acoplado à balança, com barra vertical e fixa e um esquadro sobre a cabeça formando um ângulo de $90^{\circ} \mathrm{com}$ a escala para se ter uma posição de valores (da mesma marca que a balança). A balança era aferida previamente e a cada dez medições.

Para as análises bioquímicas, foram colhidos cerca de $5 \mathrm{~mL}$ de sangue por punção venosa, de cada escolar, após jejum de 12 a 14 horas, em frascos secos para as ta- 
xas bioquímicas, os quais foram acondicionados em caixas de isopor contendo gelo reciclável, vedadas e transportadas para análise num prazo máximo de 2 horas. A coleta sangüínea foi realizada em salas previamente preparadas no interior das escolas. As amostras de sangue foram processadas e o soro imediatamente analisado em equipamento semi-automático (Biosystems ${ }^{\circledR}$ 310). O colesterol total, o HDL-colesterol e os triglicerídeos foram determinados por método colorimétrico enzimático, seguindo as instruções do fabricante (Labtest $\left.{ }^{\circledR}\right)$. O LDL-colesterol foi calculado obedecendo à fórmula proposta por Friedwald.

O perfil lipídico é definido pelas determinações do CT, HDL-c, TGL e, quando possível, do LDL-c, após jejum mínimo de 12 a 14 horas. Foi considerado como portador de dislipidemia o indivíduo que apresentasse, entre esses exames, pelo menos um valor alterado ${ }^{3}$. Os pontos de corte utilizados foram os propostos pelas normas das III Diretrizes Brasileiras sobre Dislipidemia $^{3}$, em que se têm valores desejáveis para $\mathrm{CT}<170 \mathrm{mg} / \mathrm{dL}, \mathrm{LDL}-\mathrm{c}<110 \mathrm{mg} / \mathrm{dL}$, HDL-c $\geq 35 \mathrm{mg} / \mathrm{dL}$ e TGL $\leq 130 \mathrm{mg} / \mathrm{dL}$, considerando-se a faixa etária trabalhada.

O diagnóstico do estado nutricional dos adolescentes foi realizado com base nos valores de IMC propostos pela Organização Mundial da Saúde (1995), que adota o critério de classificação percentilar do Índice de Massa Corporal (IMC), expresso $\mathrm{em} \mathrm{kg} / \mathrm{m}^{2}$, segundo idade e sexo, do padrão de referência National Health and Nutrition Examination Survey - NHANES II. Foi considerado com sobrepeso o adolescente com percentil $\geq 85^{19}$.

Para a análise descritiva dos dados utilizou-se o Epi Info, versão 3.3. Foi aplicado o teste de Mann-Whitney para verificar diferenças nas concentrações de taxas bioquímicas entre os sexos e os tipos de escola. A normalidade da distribuição das variáveis contínuas foi avaliada pelo teste de Komolgorov-Smirnov. O IMC dos estudantes foi categorizado em tercis e, através do software Stata, versão 7.0, aplicou- se o teste de Bartlett para análise de variância, enquanto o teste de Bonferroni avaliou a associação das médias das taxas bioquímicas com os diferentes tercis de IMC. Em todos os testes foi considerado o intervalo de confiança de $95 \%$ e o nível de significância de $5 \%(\mathrm{p}<0,05)$.

\section{Resultados}

Dos 180 adolescentes incluídos no estudo, $61,1 \%$ eram do sexo feminino. $O$ percentual de estudantes do ensino público foi maior $(52,8 \%)$ que o do ensino privado (47,2\%). A idade média foi de 15,3 \pm 1,0 anos, com mediana e moda iguais a 15 anos. Os valores de IMC delimitantes de cada tercil foram: 20,13; 22,43 e 29,41 para o $1^{\circ}, 2^{\circ}$ e $3^{\circ}$ tercis, respectivamente. As características antropométricas e bioquímicas dos adolescentes avaliados estão apresentadas na Tabela 1.

O percentual de sobrepeso na amostra foi de $14,4 \%$. Esta categoria do estado nutricional foi maior na escola pública $(15,8 \%)$ do que na privada $(12,9 \%)$, e nos homens $(15,7 \%)$ do que nas mulheres (13,3\%), porém não foram encontradas diferenças estatisticamente significantes para ambos os casos. Não houve registro de obesidade e o percentual de desnutrição foi inexpressivo, correspondendo a $1,7 \%$ dos casos.

As prevalências de alterações observadas nas diferentes taxas do perfil lipídico dos 180 adolescentes estão apresentadas na Tabela 2. Foram detectadas baixas prevalências para a hipercolesterolemia, assim como para o aumento do LDLcolesterol. Diferentemente, foram registradas maiores prevalências para a alteração do HDL-colesterol e dos triglicerídeos. A prevalência de dislipidemia foi de $66,7 \%$ $(\mathrm{n}=120)$.

A análise das taxas bioquímicas, separadamente, frente ao índice de massa corporal, mostrou uma associação estatisticamente significante entre IMC e colesterol total, bem como entre IMC e a fração LDLcolesterol (Tabela 3). No entanto, a asso- 
Tabela 1 - Características antropométricas e bioquímicas de escolares, Campina Grande-PB, 2004.

Table 1 - Anthropometric and biochemical characteristics of schoolchildren, Campina Grande-PB, 2004.

\begin{tabular}{lccccccc}
\hline & TOTAL & \multicolumn{2}{c}{ SEXO } & \multicolumn{3}{c}{ TIPO DE ESCOLA } \\
& & Masculino & Feminino & $\rho$ & Pública & Privada & $\rho$ \\
& $(\mathrm{n}=180)$ & $(\mathrm{n}=70)$ & $(\mathrm{n}=110)$ & & $(\mathrm{n}=95)$ & $(\mathrm{n}=85)$ & \\
\hline Idade (anos) & $15,3 \pm 1,0$ & $15,3 \pm 1,0$ & $15,3 \pm 1,0$ & $\mathbf{0 , 9 1 5}$ & $15,1 \pm 0,9$ & $15,5 \pm 1,1$ & $\mathbf{0 , 0 4 0}$ \\
Peso (Kg) & $54,9 \pm 10,3$ & $56,0 \pm 10,2$ & $54,2 \pm 10,3$ & $\mathbf{0 , 2 2 9}$ & $55,0 \pm 10,8$ & $54,9 \pm 9,7$ & $\mathbf{0 , 7 0 8}$ \\
Altura (cm) & $160,3 \pm 9,5$ & $162,2 \pm 9,5$ & $159,2 \pm 9,4$ & $\mathbf{0 , 0 6 0}$ & $161,4 \pm 9,3$ & $159,1 \pm 9,7$ & $\mathbf{0 , 1 6 0}$ \\
IMC (peso/alt $\left.{ }^{2}\right)$ & $21,2 \pm 2,7$ & $21,2 \pm 2,8$ & $21,2 \pm 2,8$ & $\mathbf{0 , 8 6 0}$ & $20,9 \pm 2,9$ & $21,5 \pm 2,5$ & $\mathbf{0 , 1 0 8}$ \\
CT* & $123,4 \pm 28,2$ & $119,6 \pm 24,5$ & $125,8 \pm 30,2$ & $\mathbf{0 , 1 2 5}$ & $116,0 \pm 14,7$ & $131,6 \pm 36,4$ & $\mathbf{0 , 0 9 6}$ \\
HDL-c* & $33,8 \pm 5,6$ & $33,6 \pm 5,7$ & $33,9 \pm 5,6$ & $\mathbf{0 , 7 9 3}$ & $33,0 \pm 4,5$ & $34,6 \pm 6,6$ & $\mathbf{0 , 0 7 8}$ \\
LDL-c* $^{*}$ & $53,7 \pm 24,4$ & $50,3 \pm 22,1$ & $55,9 \pm 25,6$ & $\mathbf{0 , 0 9 1}$ & $48,5 \pm 13,0$ & $59,6 \pm 31,9$ & $\mathbf{0 , 6 6 5}$ \\
TGL* & $108,8 \pm 21,6$ & $107,2 \pm 18,5$ & $109,7 \pm 23,4$ & $\mathbf{0 , 7 5 4}$ & $104,1 \pm 14,0$ & $114,0 \pm 27,0$ & $\mathbf{0 , 0 5 1}$ \\
\hline
\end{tabular}

Média \pm desvio padrão (Teste Kruskal Wallis/Mann Withney)

Mean \pm standard deviation (Kruskal Wallis/Mann Withney Tests)

${ }^{*} \mathrm{CT}=$ colesterol total, $\mathrm{HDL}-\mathrm{C}=$ =lipoproteína de alta densidade, $\mathrm{LDL}-\mathrm{c}=$ lipoproteína de baixa densidade, $\mathrm{TGL}=$ triglicerídeos

${ }^{*} \mathrm{CT}=$ total cholesterol, $\mathrm{HDL}=$ high-density lipoprotein, cholesterol, $\mathrm{LDL}-\mathrm{c}=$ low-density lipoprotein, cholesterol, $T G L=$ triglycerides: $\mathrm{mg} / \mathrm{dL}$

Tabela 2 - Classificação do perfil lipídico de escolares, Campina Grande-PB, 2004.

Table 2 - Classification of lipid profile in schoolchildren, Campina Grande-PB, 2004.

\begin{tabular}{|c|c|c|c|c|c|c|}
\hline \multirow{2}{*}{$\begin{array}{l}\text { TAXAS } \\
\text { BIOQUÍMICAS }\end{array}$} & \multicolumn{2}{|c|}{ Desejável } & \multicolumn{2}{|c|}{ Limítrofe } & \multicolumn{2}{|c|}{ Alterado } \\
\hline & $\mathrm{n}$ & $\%$ & $\mathrm{n}$ & $\%$ & $\mathrm{n}$ & $\%$ \\
\hline Colesterol total & 166 & 92,2 & 08 & 4,4 & 06 & 3,3 \\
\hline HDL-colesterol & 78 & 43,3 & - & - & 102 & 56,7 \\
\hline LDL-colesterol & 171 & 95,5 & 05 & 2,8 & 03 & 1,7 \\
\hline Triglicerídeos & 160 & 88,9 & - & - & 20 & 11,1 \\
\hline
\end{tabular}

Tabela 3 - Média e desvio-padrão das taxas bioquímicas, de acordo com os tercis de IMC.

Table 3 - Mean and standard deviation for biochemical indexes according to BMI tertiles.

\begin{tabular}{|c|c|c|c|c|c|c|}
\hline Variáveis & $\begin{array}{c}\text { Tercis } \\
\text { de IMC }\end{array}$ & Média $\pm d p$ & $\begin{array}{l}\rho \text { entre } \\
\text { os tercis }\end{array}$ & $\begin{array}{c}\rho 1^{\circ} \text { e } 2^{\circ} \\
\text { tercis }\end{array}$ & $\begin{array}{c}\rho 1^{\circ} \text { e } 3^{\circ} \\
\text { tercis }\end{array}$ & $\begin{array}{c}\rho 2^{\circ} \text { e } 3^{\circ} \\
\text { tercis }\end{array}$ \\
\hline \multirow[t]{3}{*}{ Colesterol total } & 10 & $112,3 \pm 13,7$ & 0,000 & 0,031 & 0,000 & 0,483 \\
\hline & $2^{\circ}$ & $125,2 \pm 33,5$ & & & & \\
\hline & $3^{\circ}$ & $132,1 \pm 29,7$ & & & & \\
\hline \multirow[t]{3}{*}{ HDL-colesterol } & 10 & $33,7 \pm 5,6$ & 0,171 & 1,00 & 0,937 & 0,184 \\
\hline & $2^{\circ}$ & $32,9 \pm 5,2$ & & & & \\
\hline & $3^{\circ}$ & $34,8 \pm 5,9$ & & & & \\
\hline \multirow[t]{3}{*}{ LDL-colesterol } & $1^{\circ}$ & $44,0 \pm 12,4$ & 0,000 & 0,021 & 0,000 & 0,735 \\
\hline & $2^{\circ}$ & $55,8 \pm 27,3$ & & & & \\
\hline & $3^{\circ}$ & $60,8 \pm 27,1$ & & & & \\
\hline \multirow[t]{3}{*}{ Triglicerídeos } & 10 & $103,8 \pm 13,5$ & 0,100 & 0,130 & 0,296 & 1,000 \\
\hline & $2^{\circ}$ & $111,8 \pm 27,0$ & & & & \\
\hline & $3^{\circ}$ & $110,4 \pm 21,4$ & & & & \\
\hline
\end{tabular}

$\rho=$ Teste de Bartlett/Bonferroni $/ \rho=$ Bartlett's test /Bonferroni 
ciação variou entre os tercis de IMC. Em ambos os casos, a significância estatística foi observada apenas do primeiro para o segundo, e do primeiro para o terceiro tercis.

Os resultados das médias de colesterol total em relação aos tercis de IMC, quando estratificados por tipo de escola e por sexo, mantiveram a significância estatística $(\mathrm{p}<0,05)$. Por outro lado, a fração LDL-c apenas manteve a significância para a escola pública e o sexo masculino. No caso do sexo feminino, observou-se uma significância marginal $(\mathrm{p}=0,052)$ e para o caso da escola privada, o valor de p observado foi de 0,07; estes valores podem ser reflexo do pequeno tamanho da amostra.

\section{Discussão}

Este estudo consiste em importante sinalizador para o fato de que as dislipidemias e o sobrepeso fazem parte de uma realidade preocupante e precisam ser mais investigados entre a população jovem do Nordeste brasileiro.

A prevalência de dislipidemia encontrada $(66,7 \%)$ foi bastante alta, consistindo em fato preocupante. Gerber e Zielinky ${ }^{20}$, em investigação realizada no sul do país com crianças e adolescentes, registraram uma prevalência de dislipidemia inferior à nossa, entre 24,0 e $33,0 \%$.

Os valores médios observados para os lipídios em diferentes estudos são bastante variáveis. Pesquisa realizada em Florianópolis, SC, com 1.053 indivíduos de 7 a 18 anos, encontraram médias de colesterol total, HDL-colesterol, triglicerídeos e LDLcolesterol de 162 $\pm 28,53 \pm 10,93 \pm 47$ e $89 \pm 24 \mathrm{mg} / \mathrm{dL}$, respectivamente. Estes valores são superiores aos encontrados no nosso estudo, com exceção dos triglicerídeos, cuja taxa média foi inferior. É importante notar, no entanto, que tanto o tamanho da amostra quanto a faixa de idade trabalhada por Giuliano et al. ${ }^{13}$, foram mais amplas.

Dentre os nossos resultados, chamou à atenção a alta prevalência de alteração nos níveis de HDL-colesterol (56,7\%). Es- tudo de método semelhante, realizado no sul do país, embora restrito a uma população de baixa renda, registrou alteração de HDL-colesterol em 17,9\% da amostra ${ }^{4}$. Em São Paulo, a prevalência de alteração verificada para essa lipoproteína foi de $13,8 \%$, em uma população de crianças e adolescentes $^{2}$. Este fato é preocupante por se tratar de populações jovens e por ser o HDL-colesterol importante fator protetor contra o desenvolvimento de doenças crônicas, particularmente da aterosclerose ${ }^{21}$.

O percentual de $11,1 \%$ de adolescentes com aumento do nível sérico dos triglicerídeos, encontrado neste estudo, foi menor do que os valores observados em estudo conduzido no Estado do Paraná, com crianças e jovens de até 19 anos de idade, no qual se registrou uma prevalência de hipertrigliceridemia de $22,5 \%{ }^{22}$. Por outro lado, nossos achados se aproximam daqueles de Romaldini et al. ${ }^{2}$, que constataram aumento dos triglicerídeos em $12,8 \%$ de 109 crianças e adolescentes com história de doença arterial coronariana prematura. Diante desta comparação é plausível admitir que a alteração de triglicerídeos registrada neste estudo seja alta, considerando-se o pressuposto de estarmos estudando uma população saudável.

Além do HDL-colesterol e dos triglicerídeos, o perfil lipídico dos estudantes deste estudo também foi alterado em função da hipercolesterolemia e do aumento do LDL-colesterol, verificados, entretanto, em menor escala. Em estudo realizado em São Paulo, com crianças e adolescentes de 2 a 19 anos, filhos de portadores de DAC, Forti et $\mathrm{al}^{23}$ encontraram valores de CT e LDLcolesterol acima dos valores considerados normais em $48,2 \%$ e $44,6 \%$ dos indivíduos, respectivamente.

Conforme foi mencionado na introdução, a relação entre dislipidemia e obesidade é referida por diversos autores ${ }^{4,24,25}$. No presente estudo não houve registro de casos de obesidade; entretanto, a prevalência de sobrepeso encontrada foi de $14,4 \%$. É importante observar que ainda 
assim foi encontrada associação estatisticamente significante dos tercis de IMC com o colesterol total e o LDL-colesterol.

O estado nutricional de adolescentes de escolas públicas e privadas do município de Campina Grande, PB, estudado por Gonzaga $^{26}$, indicou uma prevalência de sobrepeso inferior $(11,5 \%)$ à nossa para estudantes de 12 a 15 anos de idade. Estes valores foram bem superiores aos verificados, em levantamento realizado com crianças e adolescentes das regiões Sudeste e Nordeste do Brasil ${ }^{27}$, no qual foram estudadas 3.317 crianças e 3.943 adolescentes, a partir de dados da pesquisa sobre padrões de vida realizada pelo Instituto Brasileiro de Geografia e Estatística (IBGE), em
1997. A prevalência de sobrepeso nos adolescentes variou entre $1,7 \%$ no Nordeste e $4,2 \%$ no Sudeste. Frente a estes valores, é plausível dizer que nossos resultados reforçam, para a região Nordeste, a tendência ao aumento do sobrepeso, e ainda que, considerando-se o tipo de escola como uma proxy para o nível socioeconômico dos estudantes, o aumento nas prevalências da obesidade ${ }^{24,28}$ e dislipidemias ocorre em diferentes estratos sociais ${ }^{4,24}$.

Os resultados desta pesquisa confirmam uma tendência mundial e sugerem a necessidade de estudos populacionais, ou de maior abrangência institucional, para que se possa delinear as necessárias intervenções para a população alvo.

\section{Referências}

1. Santos Filho RD, Martinez TLR. Fatores de risco para doença cardiovascular: Velhos e novos fatores de risco, velhos problemas. Arq Bras Endocrinol Metab 2002; 46: $212-4$.

2. Romaldini CC, Issler H, Cardoso AL, Diament J, Forti, N. Fatores de risco para aterosclerose em crianças e adolescentes com história familiar de doença arterial coronariana prematura. Jornal de Pediatria 2004; 80(2): $135-40$.

3. Santos, DR. III Diretrizes Brasileiras sobre Dislipidemias e Diretriz de Prevenção da Aterosclerose do Departamento de Aterosclerose da Sociedade Brasileira de Cardiologia. Arq Bras Cardiol 2001; 77 (S3): 1-48.

4. Grillo LP, Crispim SP, Siebert AN, Andrade ATW, Rossi A, Campos IC. Perfil lipídico e obesidade em escolares de baixa renda. Rev Bras Epidemiol 2005; 8(1): 75-81.

5. Sposito, CA et al. IV Diretriz Brasileira sobre Dislipidemias e Prevenção da Aterosclerose Departamento de Aterosclerose da Sociedade Brasileira de Cardiologia. Arq Bras Cardiol 2007; 88(S1): 2-18.

6. Menotti A, Lanti M, Puddu PE, Kromhout D. Coronary heart disease incidence in northern and southern European population: a reanalysis of seven countries study for a European coronary risk chart. Heart 2000; 84: 238-44.

7. Castelli WP, Garrison RJ, Wilson PWF, Abbott RD, Kalousdian S, Kannel WB. Incidence of coronary heart disease and lipoprotein cholesterol levels. The Framingham Study. JAMA 1986; 256: 2835-8.

8. Gianini SD. Aterosclerose e dislipidemias. $1^{\text {a }}$ ed. São Paulo: BG Editora e Produções culturais Ltda; 1998.
9. Kannel WB, Wilson PWF. An update on coronary risk factors. Med Clin North Am 1995; 79: 951-71.

10. Tracy RE. Risk factors and atherosclerosis in youth autopsy findings of the Bogalusa Heart Study. Am J Med Sci 1995; 310 (S1): 37-41.

11. Pellanda LC, Echenique L, Barcellos LMA, Malcom GJ, Tracy RE, Strong JP. Origin of atherosclerosis in childhood and adolescence. Am J Clin Nutr 2000; 72 : 1307-15.

12. Claro RD. O mal do novo século. Rev Educação \& Família 2004; 6(1): 20-7.

13. Giuliano ICB, Coutinho MSSA, Freitas SFT, Pires MMS, Zunino JN, Ribeiro RQC. Lípides séricos em crianças e adolescentes de Florianópolis, SC - Estudo Floripa Saudável 2040. Arq Bras Cardiol 2005; 85(2): 85-91.

14. Brotons C, Ribera A, Perich RM, Abrodos D, Magaña P, Pablo S. Worldwide distribution of blood lipids and lipoproteins in childhood and adolescence: a review study. Aterosclerosis 1998; 139: 1-9.

15. Fontal MR, Espín AR. Hipercolesterolemia en la poblacion adolescente. Revista de la Faculdad de Medicina 2000; 23(1): 1-7.

16. Monteiro CA, D’A Benício MH, Conde WL, Popkin BM. Shifting obesity trends in Brazil. Eur J Clin Nutr 2000; 54: 342-6.

17. Escoda MSQ, Pinheiro DC. Repercussões nutricionais sobre o crescimento e o desenvolvimento do adolescente. III Manual de Adolescência da Sociedade Brasileira de Pediatria 1995; 1 (3). Disponível em http:/ / www.ufrnet.br/ scorpius/13-cresc\%20e\%20desenv.Htm (Acessado em: 15 de agosto de 2006) 
18. Ministério da Saúde do Brasil. Conselho Nacional de Saúde. Diretrizes e normas regulamentadoras de pesquisas envolvendo seres humanos: resolução 196/96 do Conselho Nacional de Saúde. Rio de Janeiro: Ministério da Saúde Fundação Osvaldo Cruz; 1998.

19. World Health Organization - WHO. Physical Status: the study and interpretation of anthropometry. WHO Technical Report Series n. 854. Geneva: WHO, 1995.

20. Gerber ZRS, Zielinsky P. Fatores de risco de aterosclerose na infância. Um estudo epidemiológico. Arq Bras Cardiol 1997; 69: 231-6.

21. Oliveira CL, Mello MT, Cintra IP, Fisberg M. Obesidade e síndrome metabólica na infância e adolescência. Rev Nutr 2004; 17(2): 132-8.

22. Seki M, Seki MO, Lima AD, Onishi MHO, Seki MO, Oliveira LAG. Estudo do perfil lipídico de crianças e jovens até 19 anos de idade. J Bras Patol Med Lab 2001; 37(4):247-51.

23. Forti N, Giannini D, Diament J, Issa J, Fukushima J, Bó $\mathrm{CD}$ et al. Fatores de risco para doença arterial coronariana em crianças e adolescentes filhos de coronariopatas jovens. Arq Bras Cardiol 1996; 66: 119-23.
24. Araújo F, Yamada AT, Araújo, MVM, Latorre MRDO, Mansur AJ. Perfil lipídico de indivíduos sem cardiopatia com sobrepeso e obesidade. Arq Bras Cardiol 2005; 84(5): 405-09.

25. Martins IS. Doenças cardiovasculares ateroscleróticas, dislipidemia, hipertensão, obesidade e diabetes melito em população da área metropolitana da região Sudeste do Brasil: II - Dislipidemia. Rev Saúde Pública 1996; 30(1): 82-9.

26. Gonzaga JM. Influências da atividade física na composição corporal de adolescentes. (dissertação de mestrado). Universidade Estadual da Paraíba, Campina Grande/PB, 2003.

27. Abrantes MM, Lamounier JA, Colosimo EA. Prevalência de sobrepeso e obesidade em crianças e adolescentes das regiões Sudeste e Nordeste. J Pediatr 2002; 78(4): 335-40.

28. Mendonça CP, Anjos LA. Aspectos das práticas alimentares e da atividade física como determinantes do crescimento do sobrepeso/obesidade no Brasil. Cad Saúde Pública 2004; 20(3): 291-7.

Recebido em: 08/11/06

Versão final reapresentada em: 31/07/07

Aprovado em: 13/08/07 